\title{
Negativität in der Politikberichterstattung: Deutschland, Österreich und die Schweiz im Vergleich
}

Engesser, Sven ; Esser, Frank ; Reinemann, Carsten ; Scherr, Sebastian ; Matthes, Jörg ; Wonneberger, Anke

\begin{abstract}
Negativität ist ein zentrales Konzept in der Forschung zur politischen Kommunikation, doch hinsichtlich Konzeptualisierung und Operationalisierung besteht weitgehend Uneinigkeit. Auch beruhen viele Befunde auf der Analyse von Spezialzeiten wie dem Wahlkampf. Der vorliegende Beitrag zerlegt Negativität in die vier zentralen Dimensionen Tonalität, Konflikt, Untauglichkeit und Unvorteilhaftigkeit und untersucht die Politikberichterstattung von Leitmedien gezielt in den Routineperioden zwischen Wahlkämpfen. Er zieht Vergleiche zwischen drei deutschsprachigen Ländern (Deutschland, Österreich und der Schweiz), Online- und OfflineAngeboten, vier Mediengattungen (öffentlicher und privatwirtschaftlicher Rundfunk, Qualitäts- und Boulevardzeitungen) sowie verschiedenen Darstellungsformen und Themen. Es zeigt sich, dass Medien in Österreich, online und in meinungsbetonten Darstellungsformen negativer über Politik berichten als in ihren Vergleichsgruppen. Schweizerische Medien bringen mehr positiv gehaltene Beiträge und stellen politische Akteure seltener unvorteilhaft dar. Außerdem offenbart sich entgegen den gängigen Erwartungen, dass die Boulevardpresse unter allen Mediengattungen am wenigsten negativ schreibt. Negativity is a key concept in political communication research but it still lacks a consensual definition and operationalization. Up to now, most studies on negativity have focused on single countries and single election campaign coverage. This article tackles that gap by disaggregating negativity into four main dimensions, namely tonality, conflict, incapability, and unfavorability. Comparing the three German-speaking countries Austria, Germany, and Switzerland, we investigate political news coverage of leading media outlets, particularly in the periods between election campaigns. The study sheds light on commonalities and differences between online and offline media, four media sectors (public and commercial broadcasting, as well as upmarket and mass-market press), and shows that the media in Austria, online outlets, and opinionated news are more negative in tone than their comparison groups. By contrast, the Swiss media publish more positive news and portray political actors in less gloomy ways. Finally, we reveal that, in contrast to common expectations, the mass-market press provides political news with the least negative touch.
\end{abstract}

DOI: https://doi.org/10.5771/1615-634x-2014-4-588

Posted at the Zurich Open Repository and Archive, University of Zurich

ZORA URL: https://doi.org/10.5167/uzh-103957

Journal Article

Published Version

Originally published at:

Engesser, Sven; Esser, Frank; Reinemann, Carsten; Scherr, Sebastian; Matthes, Jörg; Wonneberger, Anke (2014). Negativität in der Politikberichterstattung: Deutschland, Österreich und die Schweiz im Vergleich. Medien Kommunikationswissenschaft, 62(4):588-605.

DOI: https://doi.org/10.5771/1615-634x-2014-4-588 


\title{
Negativität in der Politikberichterstattung
}

\author{
Deutschland, Österreich und die Schweiz im Vergleich
}

\section{Sven Engesser / Frank Esser / Carsten Reinemann / Sebastian Scherr / Jörg Matthes / Anke Wonneberger}

Negativität ist ein zentrales Konzept in der Forschung zur politischen Kommunikation, doch hinsichtlich Konzeptualisierung und Operationalisierung besteht weitgehend Uneinigkeit. Auch beruben viele Befunde auf der Analyse von Spezialzeiten wie dem Wahlkampf. Der vorliegende Beitrag zerlegt Negativität in die vier zentralen Dimensionen Tonalität, Konflikt, Untanglichkeit und Unvorteilhaftigkeit und untersucht die Politikberichterstattung von Leitmedien gezielt in den Routineperioden zwischen Wablkämpfen. Er zieht Vergleiche zwischen drei deutschsprachigen Ländern (Deutschland, Österreich und der Schweiz), Online- und Offline-Angeboten, vier Mediengattungen (öffentlicher und privatwirtschaftlicher Rundfunk, Qualitäts- und Boulevardzeitungen) sowie verschiedenen Darstellungsformen und Themen. Es zeigt sich, dass Medien in Österreich, online und in meinungsbetonten Darstellungsformen negativer über Politik berichten als in ibren Vergleichsgruppen. Schweizerische Medien bringen mebr positiv gehaltene Beiträge und stellen politische Akteure seltener unvorteilhaft dar. Außerdem offenbart sich entgegen den gängigen Erwartungen, dass die Boulevardpresse unter allen Mediengattungen am wenigsten negativ schreibt.

Schlüsselwörter: Negativität, Konflikt, Tonalität, politische Kommunikation, internationaler Vergleich, Inhaltsanalyse

\section{Einleitung}

Kaum ein Kritikpunkt wird den Massenmedien mit größerer Vehemenz und Regelmäßigkeit entgegengebracht als der Vorwurf der Schwarzmalerei (z. B. Patterson 1996, 2002). Wenn sich Politiker und andere Prominente in diese Richtung äußern, ist der Anlass fast immer eine unsystematische Beobachtung, z. B. der reißerischen Titelseite einer Boulevardzeitung (Kepplinger 2010: 175). In der Kommunikationswissenschaft beruhen die meisten Schlussfolgerungen zur Negativität der Medien auf empirischen Analysen von Spezialfällen, wie der relativ stark polarisierten Berichterstattung im Wahlkampf (z. B. Esser \& Hemmer 2008; Reinemann et al. 2013; Walter \& Vliegenhart 2010; Weiß 1982). Die Nachrichtenwertforschung nimmt zwar durchaus Routineperioden in den Blick, behandelt Negativität jedoch in der Regel nur am Rande (z. B. Eilders 1997; Ruhrmann et al. 2003). Ein Forschungszweig, der sich gezielt der Negativität widmet und diese außerhalb von Wahlkampagnen untersucht, ist derzeit noch in der Entwicklung begriffen (z. B. Maurer 2003; Wolling 1999). Auch international vergleichende Arbeiten liegen bisher kaum vor (z. B. Plasser et al. 2009; Magin 2012).

Negativität muss nicht zwangsläufig etwas Schlechtes sein. Der Mensch ist sogar besonders empfänglich für negative Nachrichten. In der Psychologie spricht man vom „Negativity Bias“, das in zahlreichen empirischen Studien bestätigt wurde (z. B. Kahneman \& Tversky 1979; im Überblick: Baumeister et al. 2001). Es hat sich offenbar als evolutionärer Vorteil erwiesen, Berichten über Katastrophen, Kriege, Unfälle und Krankheiten erhöhte Aufmerksamkeit zu schenken, um Risiken und Gefahren zu vermeiden. Außerdem impliziert Negativität häufig eine Abweichung vom Status Quo 
(Shoemaker \& Cohen 2006). Daher hat sie sich mit der Zeit als dauerhafte Wahrnehmungskategorie etabliert. Dies schlägt sich im klassischen Nachrichtenfaktor „Negativismus“ (Galtung \& Ruge 1965, O’Neill \& Harcup 2009) und den verwandten Faktoren „Kontroverse“ und „Schaden“ nieder (z. B. Eilders 1997; Ruhrmann et al. 2003). Negativität kann also ein nützliches Warnsignal für Entwicklungen sein, die unsere Aufmerksamkeit verdienen.

Auch aus demokratietheoretischer Perspektive erfüllt Negativität einen Zweck (Westen 2007). Wenn sie mit der Kritik an Missständen, der Kontrolle der Regierung und der Aufdeckung von politischen Skandalen einhergeht, hat sie durchaus eine funktionale Komponente. Negativität kann bei den Bürgern gesunde Skepsis erzeugen, sie bei der Ausübung ihrer Rechte und Pflichten unterstützen und zu informierten Entscheidungen animieren (Norris 2000).

Negativität kann jedoch auch eine destruktive Qualität aufweisen. Zum einen kann sie lediglich ein Ausdruck von Sensationalismus, Effekthascherei und Schadenfreude sein. Zum anderen kann sich in ihr eine pauschal negative Grundhaltung der Medien gegenüber den Mächtigen in der Gesellschaft manifestieren, die man als „adversarial spirit“ (Bennett 2012) oder „Nachrichtenideologie“ (Kepplinger \& Weissbecker 1991) beschreiben kann. Wenn Negativität in dieser Form auftritt oder im Übermaß vorkommt, kann sie zu Zynismus und Politikverdrossenheit führen (Bennett 2012).

Angesichts dieses stark normativen Gehalts ist es nicht verwunderlich, dass Negativität in der Forschung zur politischen Kommunikation ein zentrales Konzept darstellt. Allerdings besteht bezüglich ihrer Konzeptualisierung (Kleinnijenhuis 2008) und Operationalisierung (Lengauer et al. 2012; Ridout \& Franz 2008) noch weitgehend Uneinigkeit. Auch widmen sich viele empirische Untersuchungen der Negativität nur sporadisch oder am Rande. Es fehlen zudem Vergleiche über Länder und Mediengattungen hinweg.

Der folgende Beitrag ist bestrebt, diese Forschungslücke zu schließen. Er greift den Vorschlag zur Operationalisierung von Negativität von Lengauer et al. (2012) auf und testet diesen empirisch. Die Politikberichterstattung von Leitmedien wird gezielt in den Routineperioden zwischen Wahlkämpfen untersucht, um die Befunde stärker als bisher verallgemeinern zu können. Zur zusätzlichen Generalisierung und Kontextualisierung wird ein Vergleich zwischen Deutschland, Österreich und der Schweiz vorgenommen. Der internationale Vergleich hat sich in der Kommunikationswissenschaft als Forschungsstrategie bewährt und bietet zahlreiche Vorteile (Blumler \& Gurevitch 1995; Esser \& Pfetsch 2003; Esser \& Hanitzsch 2012). Dazu gehört in erster Linie die Möglichkeit, empirische Befunde zu kontextualisieren und kommunikationswissenschaftliche Theorien zu generalisieren. Auf diese Weise kann dem „naiven Universalismus“ vorgebeugt werden, nach dem „eine im eigenen Land gefundene Gesetzmäßigkeit auch in anderen Ländern gilt" (Esser \& Pfetsch 2003: 437).

Im vorliegenden Beitrag folgt die Auswahl der Untersuchungsländer dem Most Similar Systems Design. Dabei sollen sich die Mediensysteme in möglichst wenigen Aspekten unterscheiden, damit Unterschiede im Medieninhalt genau auf diese zurückgeführt werden können (Przeworski \& Teune 1970; Wirth \& Kolb 2003). Obwohl sich Deutschland, Österreich und die Schweiz global gesehen sehr stark ähneln, weichen sie bezüglich des politischen Systems, der demokratischen Qualität und der journalistischen Kultur voneinander $\mathrm{ab}$ (siehe Abschnitt 2.1). Darüber hinaus zieht der Beitrag Vergleiche zwischen Online- und Offline-Angeboten, vier Mediengattungen (öffentlicher und privatwirtschaftlicher Rundfunk, Qualitäts- und Boulevardzeitungen) sowie verschiedenen Darstellungsformen und Themen. 


\section{Hypothesen und Forschungsfragen}

Die bisherige Forschung zur Negativität in der Politikberichterstattung konzentriert sich in erster Linie auf drei Bereiche: Erstens wurde untersucht, wie negativ die Medien über Wahlkämpfe berichten und inwiefern diese Negativität als Indikator für Medialisierung gedeutet werden kann (z. B. Esser \& Hemmer 2008; Plasser et al. 2009; Reinemann et al. 2013). Zweitens wurde Negativität als mögliche Ursache von Politikverdrossenheit verstanden und auch außerhalb von Wahlkämpfen erforscht (z. B. Maurer 2003; Wolling 1999). Drittens wurde Negativität im Rahmen der Nachrichtenwertforschung analysiert (z. B. Eilders 1997; Ruhrmann et al. 2003; im Überblick: O’Neill \& Harcup 2009). Aus dem Literaturkorpus dieser drei Forschungszweige und ausgewählten Erkenntnissen der international vergleichenden Politikwissenschaft und Kommunikationsforschung werden im Folgenden Hypothesen und Forschungsfragen abgeleitet (für eine allgemeinere Literaturübersicht siehe: Lengauer et al. 2012). Diese sind nach Länder-, Medien- und Beitragsebene geordnet.

\subsection{Länderebene}

Auf Länderebene unterscheiden sich Deutschland, Österreich und die Schweiz hinsichtlich ihres politischen Systems, der demokratischen Qualität und der journalistischen Kultur. Als Ausgangspunkt für die Illustration dieser Unterschiede bietet sich Lijpharts (2012) vielzitierte Einordnung in Konsensdemokratien und Mehrheitsdemokratien an. Danach gilt die Schweiz als „Prototyp“ (S. 245) der Konsensdemokratie, in erster Linie aufgrund des einzigartigen Konkordanzprinzips. Deutschland rangiert zwar dahinter, trägt jedoch durch den ausgeprägten Föderalismus (S. 178) und die dezidierte Normenkontrolle (S. 215) ebenfalls stark konsensuale Züge. Österreich ist im Vergleich dazu eher mehrheitsdemokratisch ausgerichtet, was sich z. B. in stabileren Regierungskabinetten (S. 120) und einer schwächeren zweiten Kammer des Parlaments niederschlägt (S. 199). Da Konsensdemokratien bei einer Reihe von Kriterien demokratischer Qualität (z. B. Gleichberechtigung der Geschlechter, ausgeglichene Einkommensverteilung und hohe Wahlbeteiligung) besser abschneiden als Mehrheitsdemokratien, bezeichnet Lijphart sie insgesamt als „freundlicher und sanfter“ (S. 287). Nach Strömbäck und Kaid (2008) ist davon auszugehen, dass sich derartige Eigenschaften politischer Systeme nicht nur in der demokratischen Qualität, sondern auch in der öffentlichen Kommunikation niederschlagen und demnach Konsensdemokratien weniger negativ, kontrovers und unvorteilhaft berichten als Mehrheitsdemokratien.

Zu Lijpharts (2012) Einordnung passt, dass Österreich deutlich schlechter im Corruption Perceptions Index von Transparency International (2012) abschneidet (Platz 25) als Deutschland (Platz 13) oder die Schweiz (Platz 6). Auch der Anteil „unzufriedener Demokraten“ (Klingemann 2013: 46) an der Gesamtbevölkerung fällt in Österreich $(34,3 \%)$ signifikant größer aus als in Deutschland (30,6 \%) und der Schweiz (17,8 \%).

Die Unterschiede beim politischen System und der demokratischen Qualität scheinen sich auf die journalistische Kultur auszuwirken. So geht es Journalisten in Österreich häufiger darum, Kritik an Missständen zu üben (75\%) als ihren Kollegen in Deutschland (58\%) (Kaltenbrunner et al. 2008: 33). Dagegen betrachten sich Journalisten in der Schweiz seltener als Watchdog der Regierung und der wirtschaftlichen Eliten und sind häufiger bereit, diese in einem positiven Licht darzustellen, als ihre Kollegen in Deutschland und Österreich (Hanitzsch et al. 2011:292). Hinweise dafür, dass sich diese Einflüsse in den Medieninhalten widerspiegeln, findet Magin (2012) im Ländervergleich zwischen Deutschland und Österreich. Sie stellt explizit fest: „Die österreichischen Zeitungen be- 
richten negativer als die deutschen“ (S. 223). Daher lassen sich aus den gesammelten Befunden folgende Hypothesen ableiten:

\section{H1 Medien in Österreich berichten negativer über Politik als Medien in Deutschland und der Schweiz. \\ H2 Medien in der Schweiz berichten weniger negativ über Politik als Medien in Deutschland und Österreich.}

\subsection{Medienebene}

Auf Medienebene lassen sich Online- und Offlineangebote, öffentliches Fernsehen und Privatfernsehen sowie Qualitäts- und Boulevardpresse voneinander abgrenzen. Quandt (2008: 150) sowie Powers und Benson (2014: 258) stellen fest, dass Onlinemedien in Europa Negativnachrichten stärker hervorheben als die zugehörigen Offline-Ausgaben. Als Erklärung dafür gilt der Hang von Onlinemedien, bestehende Nachrichten offensiv weiterzudrehen - und zwar mit geringfügiger Zusatzrecherche und hektischem Blick auf die Wettbewerber. Allerdings räumt Quandt (2008) ein, dass in diesem Zusammenhang „spezifische Anschlussforschung“ erforderlich wäre (S. 151). Oschatz et al. (2014) bestätigen eine hohe Onlinepräferenz für Negativität bei den von ihnen untersuchten $\mathrm{Me}$ dien, außer beim gedruckten Spiegel im Vergleich zu Spiegel Online. Hier enthielt die Printversion mehr negative Akteursdarstellungen als die Onlinevariante (33\% vs. $23 \%$ ). Für diese Autoren liegen die Unterschiede weniger im Produktionsprozess begründet, da die Nähe zwischen den Onlineangeboten und ihren Muttermedien häufig sehr hoch ist. Vielmehr könnten Onlinemedien mehr Negativität einsetzen, um in der Pull-Umgebung des Internets höhere Aufmerksamkeit zu generieren. Es blieb zwar bisher offen, ob sich diese Ergebnisse auf Österreich und die Schweiz übertragen lassen. Dennoch erscheint die Formulierung der folgenden Hypothese gerechtfertigt:

\section{H3 Onlinemedien berichten negativer als Offlinemedien.}

Plasser et al. (2009: 192) können zeigen, dass das öffentliche Fernsehen in Deutschland, Österreich und Italien positiver über Wahlkämpfe berichtet als das Privatfernsehen. In die gleiche Richtung gehen Befunde von Ruhrmann et al. (2003: 82), wonach in der deutschen Politikberichterstattung der Nachrichtenfaktor „Kontroverse“ im öffentlichrechtlichen Fernsehen weniger häufig Verwendung findet (61 bis $67 \%$ ) als im Privatfernsehen (71 bis 73 \%). Auch Maurer (2003) findet heraus, dass in Deutschland Privatsender negativer berichten als öffentlich-rechtliche Sender (wenngleich diese Unterschiede im Zeitverlauf verschwimmen). Zum einen lassen sich diese Befunde damit begründen, dass sich Journalisten des öffentlichen Fernsehens stärker der Ausgewogenheit verpflichtet fühlen als ihre Kollegen beim Privatfernsehen. Zudem wird vermutet, dass das öffentliche Fernsehen den politischen Institutionen kulturell näher steht und sie daher seltener missbilligend beleuchtet (Hanitzsch \& Berganza 2012). Zum anderen lässt sich argumentieren, dass das Privatfernsehen aufgrund seiner stärker ökonomischen Orientierung mittels Sensationalismus mehr Aufmerksamkeit beim Publikum generieren möchte. Allerdings weist Maurer (2003) darauf hin, dass es auch auf den Sendungstyp ankommt: So berichten meinungsbetonte Formate des öffentlich-rechtlichen Fernsehens nicht nur negativer als öffentlich-rechtliche Nachrichtensendungen, sondern auch als Magazinsendungen im Privatfernsehen. Dennoch lässt sich folgende Hypothese aufstellen:

H4 Öffentliches Fernseben berichtet weniger negativ als Privatfernseben. 
Die Akteure im Bundestagswahlkampf 1980 wurden in Qualitätszeitungen negativer dargestellt als im öffentlich-rechtlichen Fernsehen (Weiß 1982). Auch Esser und Hemmer (2008: 298) beobachten bei deutschen Wahlkämpfen mehr Negativität in der Presse als im Fernsehen. Dagegen zeigen Walter und Vliegenhart (2010: 451), dass im Vorfeld der niederländischen Parlamentswahlen 2006 Fernsehdebatten negativer gehalten waren als Zeitungsberichte. Maurer und Reinemann (2006: 135) differenzieren, dass politische Magazine im Fernsehen durchschnittlich (relative Tendenz) negativer berichten, Tageszeitungen jedoch negativer berichten, wenn man den Saldo aus positiven und negativen Beiträgen (absolute Tendenz) heranzieht (Maurer 2003: 131; Maurer \& Reinemann 2006: 135). Zwischen Qualitäts- und Boulevardzeitungen lassen sich trotz zum Teil ausgeprägter redaktioneller Linien in zahlreichen deutschen Wahlkampfstudien auf den ersten Blick nur wenige systematische Differenzen in puncto Negativität ausmachen, etwa bei der Bewertung von Parteien, Koalitionen und Kanzlerkandidaten (z. B. Reinemann et al. 2013; im Überblick: Maurer \& Reinemann 2006: 133-139). Die Bild-Zeitung nimmt eine Sonderrolle ein, denn das Boulevardblatt berichtet in seiner alltäglichen politischen Berichterstattung insgesamt positiv - dies trifft jedoch für die Skandalberichterstattung keineswegs zu (Maurer 2003; Wolling 1999). Eilders (1997) findet die Nachrichtenfaktoren „Kontroverse“ und „Schaden“ häufiger in der Politikberichterstattung der überregionalen Abonnementzeitungen als der Straßenverkaufszeitungen, was ihrer Meinung nach dem „gängigen Klischee von Qualitäts- und Boulevardberichterstattung“ (S. 185) widerspricht. Aufgrund dieser recht gemischten Befunde wird von der Aufstellung einer Hypothese Abstand genommen und stattdessen folgende Forschungsfrage formuliert:

FF1 Welche Negativitätsunterschiede bestehen zwischen Qualitäts- und Boulevardpresse?

\subsection{Beitragsebene}

Auf Beitragsebene sind die Unterschiede zwischen den verschiedenen Darstellungsformen und Themen angesiedelt. Obwohl bisher kaum systematische Forschungsergebnisse zur Negativität bei meinungsbetonten (z. B. Kommentar und Leitartikel), tatsachenbetonten (z. B. Bericht und Meldung) und erzählenden (z. B. Reportage und Portrait) Darstellungsformen vorliegen, erscheint es plausibel, dass die Formen der ersten Kategorie negativer gehalten sind als die Formen der beiden übrigen Kategorien. Dies hängt in erster Linie damit zusammen, dass meinungsbetonte Darstellungsformen nicht dem Objektivitätsideal unterliegen, sondern als Forum für die Kritik an Missständen und die Kontrolle der Regierung dienen (Schönbach 1977). Daher liegt folgende Hypothese auf der Hand:

\section{H5 Meinungsbetonte Darstellungsformen sind negativer gehalten als die übrigen For- men.}

Der thematische Kontext dürfte ebenfalls Einfluss darauf nehmen, inwieweit Medienbeiträge eine negative Rahmung erhalten. Die Charakteristika von Themen und zugrundeliegenden Ereignissen bestimmen den journalistischen Umgang mit ihnen (de Swert et al. 2013). Daher erwarten wir, dass Themen, die eine Verbindung zu negativ konnotierten Ereignissen haben oder entsprechende Priming-Effekte ausstrahlen - wie etwa Kriminalität, Korruption oder Immigration - auch negativer berichtet werden. Da wir diese Plausibilitätsannahme jedoch nicht mit Literatur abstützen können, formulieren wir eine offene Forschungsfrage:

\section{FF2 Welche Negativitätsunterschiede bestehen zwischen verschiedenen Themen?}




\section{Methode}

Zur Überprüfung der Hypothesen und Beantwortung der Forschungsfragen wurde eine standardisierte Inhaltsanalyse der Politikberichterstattung während der Routineperioden außerhalb der Wahlkämpfe in Deutschland, Österreich und der Schweiz durchgeführt. Im Folgenden werden Operationalisierung, Stichprobe und die Details der Durchführung kurz vorgestellt.

\subsection{Operationalisierung}

Die Operationalisierung der Negativität lehnte sich an den Vorschlag von Lengauer et al. (2012) an und basierte auf den vier Dimensionen Tonalität, Konflikt, Untauglichkeit und Unvorteilhaftigkeit. ${ }^{1}$ Tonalität erfasste auf einer Skala von -1 (= positiv) über 0 (= ambivalent/neutral) zu 1 (= negativ) den Gesamteindruck des Beitrags. Es wurde codiert, ob politische Ereignisse als Misserfolg, Problem, Schaden oder Enttäuschung dargestellt wurden oder als Erfolg, Nutzen oder Lösung. Die Konfliktdimension bewegte sich auf einer Skala von -1 (= Konsens) über 0 (= ambivalent/neutral) zu 1 (= Konflikt). Dabei wurde Konflikt verstanden als Uneinigkeit, Kontroverse oder Streit und Konsens als Einigung, Kompromiss oder Zusammenarbeit. Ausschlaggebend für die Codierung war, dass bei einem bestimmten Sachverhalt mindestens zwei verschiedene Konfliktparteien oder inhaltliche Positionen existierten.

Untauglichkeit maß auf einer Skala von -1 (= Tauglichkeit) über 0 (= ambivalent/ neutral) zu 1 (= Untauglichkeit), ob zum Ausdruck gebracht wurde, dass eine Person für ein politisches Amt oder Mandat geeignet war oder nicht. Unvorteilhaftigkeit erfasste auf einer Skala von -1 (= vorteilhaft) über 0 (= ambivalent/neutral) zu 1 (= unvorteilhaft), ob ein Politiker durch Fakten, Zitate und Bewertungen in positivem oder negativem Licht dargestellt wurde. Während die ersten drei Dimensionen auf Beitragsebene gemessen wurden, wurde die letzte für die fünf wichtigsten Akteure im Beitrag erhoben und durch Berechnung des arithmetischen Mittels auf Beitragsebene aggregiert. Die Erhebungseinheit war also in drei Fällen der Beitrag und einmal der Akteur innerhalb des Beitrags.

Alle vier Dimensionen korrelieren relativ stark und statistisch signifikant miteinander (siehe Tabelle 1). ${ }^{2}$ Die Koeffizienten für die Korrelationen zwischen den drei beitragsbezogenen Dimensionen Tonalität, Konflikt und Untauglichkeit fallen zwar etwas höher aus als für die Korrelationen mit der akteursbezogenen Dimension Unvorteilhaftigkeit. Dennoch ergibt eine Hauptkomponentenanalyse nur eine einzige Komponente mit einem Eigenwert von $\lambda=2,45$, die $61 \%$ der Gesamtvarianz erklärt. Eine hypothetische zweite Komponente käme lediglich auf einen Eigenwert von $\lambda=0,69$ und erklärte $17 \%$ Varianz. Aus diesem Grund wurden die vier Dimensionen zu einem Negativitätsindex (arithmetisches Mittel) zusammengefasst, der eine akzeptable interne Konsistenz (Cronbachs $\alpha=, 78$ ) aufweist.

1 Lengauer et al. (2012) schlagen zwar noch eine fünfte Dimension der Negativität vor - pessimistischer Ausblick - doch diese wurde in der bisherigen Forschung kaum verwendet und hat sich während des Pretests der vorliegenden Untersuchung als nicht reliabel messbar erwiesen. Daher wurde sie verworfen.

2 Da keine plausiblen Einwände dagegen vorlagen und dies in der Nachrichtenforschung eine gängige Praxis darstellt, wurde bei den zur Messung der Dimensionen verwendeten Skalen Äquidistanz unterstellt, sodass auch Analysen, die Intervallskalenqualität erforderten, sinnvoll interpretierbar waren. 
Tabelle 1: Dimensionen der Negativität

\begin{tabular}{lccc} 
Dimension & Konflikt & Untauglichkeit & Unvorteilhaftigkeit \\
\hline Tonalität &, $57^{* *}$ &, $62^{* *}$ &, $44^{* *}$ \\
Konflikt & &, $49^{* *}$ &, $34^{* *}$ \\
Untauglichkeit & &, $40^{* *}$ \\
\hline
\end{tabular}

Anmerkung: $N_{\text {Min }}=1346$; Werte sind Pearsons Korrelationskoeffizienten; $*$ Markierte Koeffizienten sind signifikant $(p<, 01)$

\subsection{Stichprobe}

In jedem der drei Untersuchungsländer Deutschland, Österreich und der Schweiz wurden zehn Leitmedien ausgewählt: zwei Qualitätszeitungen (jeweils eine politisch mitterechts und mitte-links gerichtet), die am weitesten verbreitete Boulevardzeitung, jeweils die beliebteste Hauptnachrichtensendung des öffentlichen Fernsehens und des Privatfernsehens sowie die Webangebote all dieser Medien (siehe Tabelle 2). Da auf dem Webangebot von TeleZüri keine nennenswerten redaktionellen Inhalte zu finden waren, wurde es durch die Website des größten kommerziellen Radiosenders der Schweiz, Radio 24, ersetzt.

\section{Tabelle 2: Zusammensetzung der Stichprobe}

\begin{tabular}{|c|c|c|c|c|}
\hline \multicolumn{2}{|c|}{ Mediengattung } & \multirow{3}{*}{$\begin{array}{l}\text { Deutschland } \\
\text { Frankfurter Allgemeine } \\
\text { Zeitung } \\
\text { Süddeutsche Zeitung }\end{array}$} & \multirow{3}{*}{$\begin{array}{l}\text { Österreich } \\
\text { Die Presse } \\
\text { Der Standard }\end{array}$} & \multirow{3}{*}{$\begin{array}{l}\text { Schweiz } \\
\begin{array}{l}\text { Neue Zürcher Zeitung } \\
\text { Tagesanzeiger }\end{array}\end{array}$} \\
\hline & Oualität & & & \\
\hline Presse & & & & \\
\hline & Boulevard & Bild & Kronen Zeitung & Blick \\
\hline \multirow{2}{*}{ TV } & Öffentlich & $\begin{array}{l}\text { ARD, Tagesschau, } \\
\text { 20:00-20:15 }\end{array}$ & $\begin{array}{l}\text { ORF1, Zeit im Bild, } \\
\text { 20:00-20:35 }\end{array}$ & $\begin{array}{l}\text { SF1, Tagesschau, } \\
\text { 19:30-20:00 }\end{array}$ \\
\hline & Privat & $\begin{array}{l}\text { RTL, RTL Aktuell, } \\
\text { 18:45-19:10 }\end{array}$ & $\begin{array}{l}\text { ATV, ATV Aktuell, } \\
\text { 19:20-19:35 }\end{array}$ & $\begin{array}{l}\text { TeleZüri, ZüriNews, } \\
\text { 18:00-18:15 }\end{array}$ \\
\hline \multirow{2}{*}{ Web } & Presse & $\begin{array}{l}\text { www.faz.de } \\
\text { www.sueddeutsche.de } \\
\text { www.bild.de }\end{array}$ & $\begin{array}{l}\text { www.diepresse.at } \\
\text { www.derstandard.at } \\
\text { www.krone.at }\end{array}$ & $\begin{array}{l}\text { www.nzz.ch } \\
\text { www.tagesanzeiger.ch } \\
\text { www.blick.ch }\end{array}$ \\
\hline & Rundfunk & $\begin{array}{l}\text { www.tagesschau.de } \\
\text { www.rtl.de/cms/news/rtl- } \\
\text { aktuell.html }\end{array}$ & $\begin{array}{l}\text { www.news.orf.at } \\
\text { www.kurier.at }\end{array}$ & $\begin{array}{l}\text { www.tagesschau.sf.tv } \\
\text { www.radio24.ch }\end{array}$ \\
\hline
\end{tabular}

\subsection{Durchfübrung}

Das Universum des Untersuchungsmaterials bestand aus allen Beiträgen, die mindestens eine Länge von zwei Sätzen aufwiesen und einen inländischen politischen Akteur erwähnten ${ }^{3}$. Die Beiträge wurden während zweier künstlicher Wochen zwischen dem 15. April und dem 15. Juli 2012 archiviert. Die Webangebote wurden jeweils zwischen

3 Die relevanten politischen Akteure wurden durch qualitative Vorstudien ermittelt und im Codebuch festgehalten. Dabei handelte es sich überwiegend um Politiker, Parteien oder Regierungen. 
14 und 16 Uhr Ortszeit mit HTTrack heruntergeladen. Für jede Tageszeitung und Nachrichtensendung wurden pro Tag fünf Beiträge nach dem Zufallsprinzip bestimmt und in die Stichprobe aufgenommen, für jedes Webangebot wurden pro Tag drei Beiträge ausgewählt. In jedem Land wurden die Daten von zwei sorgfältig geschulten Codierern erhoben. Diese führten vor (und teilweise nach) der Erhebung Reliabilitätstests durch, welche zufriedenstellende Ergebnisse erzielten (siehe Tabelle 3).

Tabelle 3: Intranationale Reliabilität nach Land

\begin{tabular}{|c|c|c|c|c|c|c|c|c|}
\hline \multirow{2}{*}{ Dimension } & \multicolumn{3}{|c|}{ Deutschland } & \multirow{2}{*}{$\begin{array}{c}\begin{array}{c}\text { Öster- } \\
\text { reich }\end{array} \\
\text { Ex-ante } \\
N=32\end{array}$} & \multicolumn{3}{|c|}{ Schweiz } & \multirow[t]{2}{*}{ Gesamt } \\
\hline & $\begin{array}{l}\text { Ex-ante } \\
N=20\end{array}$ & $\begin{array}{c}\text { Ex-post } \\
N=30\end{array}$ & $\begin{array}{l}\text { Gesamt } \\
N=50\end{array}$ & & $\begin{array}{l}\text { Ex-ante } \\
N=23\end{array}$ & $\begin{array}{l}\text { Ex-pos } \\
N=28\end{array}$ & $\begin{array}{l}\text { Gesamt } \\
N=51\end{array}$ & \\
\hline Tonalität & ,75 & ,95 & ,87 & ,81 &, 32 &, 84 & ,61 & ,76 \\
\hline Konflikt & ,70 & ,89 & ,81 & ,81 & ,74 & 1,00 & ,88 & ,84 \\
\hline Untauglichkeit & ,80 & 1,00 & ,92 & ,71 & ,65 & ,90 & ,79 & ,81 \\
\hline Unvorteilhaftigkeit & ,81 & ,95 & ,89 & ,85 & ,78 & ,93 & ,86 & ,87 \\
\hline Darstellungsform & ,72 & 1,00 & ,88 & ,93 & 1,00 & ,94 & ,97 & ,93 \\
\hline Thema & ,71 & ,96 & ,86 & ,94 & ,76 & ,94 & ,96 & ,89 \\
\hline Gesamt & ,77 & ,95 & ,88 & 80 & ,62 & ,92 & ,78 & ,85 \\
\hline
\end{tabular}

Anmerkung: Werte sind Krippendorff's $\alpha$

\section{Ergebnisse}

Der Negativitätsindex für alle 1366 Beiträge liegt bei durchschnittlich 0,20. Die Konfliktdimension zieht den Indexwert am stärksten nach oben $(M=0,36)$ und die Unvorteilhaftigkeit am stärksten nach unten $(M=0,01)$. Deutlich mehr als die Hälfte $(57,5 \%)$ der Beiträge sind eher negativ gehalten (Index $>0)$ und rund ein Viertel $(25,2 \%)$ eher positiv (Index <0). Ein Anteil von 17,3\% ist exakt neutral oder ausgewogen (Index =0).

\subsection{Unterschiede zwischen Deutschland, Österreich und der Schweiz}

Die Medien in Österreich berichten durchschnittlich signifikant negativer über Politik als die Medien in Deutschland und der Schweiz. Dieses Muster zieht sich durch alle vier Dimensionen des Index (siehe Tabelle 4). Dadurch bestätigt sich Hypothese 1 umfassend. Betrachtet man die Mittelwerte des Negativitätsindex (erste Zeile in Tabelle 4) liegen die deutschen und schweizerischen Medien vollkommen gleichauf. Nur die Unvorteilhaftigkeitsdimension ist in der Schweiz signifikant am schwächsten ausgeprägt. Die Tonalität erreicht in Deutschland die signifikant niedrigsten Werte. Daher bestätigt sich Hypothese 2 vorerst nicht.

Wirft man jedoch einen Blick auf die Verteilung des Negativitätsindex (siehe Tabelle 5), liegt der Anteil der eher positiv gehaltenen Beiträge in der Schweiz bei rund einem Drittel $(33,5 \%)$ und ist damit signifikant größer als in Deutschland $(25,1 \%)$ und Österreich $(18,4 \%)$. Demzufolge berichten schweizerische Medien zwar durchschnittlich nicht weniger negativ als deutsche Medien, bringen jedoch mehr positiv gehaltene Beiträge. Wird Hypothese 2 dementsprechend modifiziert, bestätigt sie sich zumindest teilweise. 
Tabelle 4: Mittelwerte der Negativität nach Land

\begin{tabular}{|c|c|c|c|c|c|c|}
\hline \multicolumn{2}{|c|}{ Negativität } & $\begin{array}{l}\text { Deutsch- } \\
\text { land } \\
(n=498)\end{array}$ & $\begin{array}{c}\text { Öster- } \\
\text { reich } \\
(n=477)\end{array}$ & $\begin{array}{l}\text { Schweiz } \\
(\mathrm{n}=391)\end{array}$ & $\begin{array}{c}\text { Gesamt } \\
(\mathrm{N}=1366)\end{array}$ & $\mathrm{F}$ \\
\hline \multicolumn{2}{|l|}{ Index } & $0,13^{a}$ & $0,33^{b}$ & $0,13^{a}$ & 0,20 & $\begin{array}{c}F(2,1363)=32,29, p<, \\
001\end{array}$ \\
\hline \multirow{4}{*}{$\begin{array}{l}\text { Dimen- } \\
\text { sion }\end{array}$} & Tonalität & $0,11^{a}$ & $0,38^{b}$ & $0,21^{\mathrm{c}}$ & 0,23 & \multirow{4}{*}{$\begin{array}{c}F(2,1363)=21,66, p<, \\
001 \\
F(2,1363)=20,10, p<, \\
001 \\
F(2,1363)=16,15, p<, \\
001 \\
F(2,1343)=46,37, p<, \\
001\end{array}$} \\
\hline & Konflikt & $0,28^{a}$ & $0,51^{b}$ & $0,29^{a}$ & 0,36 & \\
\hline & Untauglichkeit & $0,10^{a}$ & $0,30^{b}$ & $0,16^{a}$ & 0,19 & \\
\hline & $\begin{array}{l}\text { Unvorteilhaftig- } \\
\text { keit }\end{array}$ & $0,01^{a}$ & $0,12^{\mathrm{b}}$ & $-0,13^{c}$ & 0,01 & \\
\hline
\end{tabular}

Anmerkung: Skala von -1 (= positiv) über 0 (= ambivalent/neutral) zu 1 (= negativ); Werte mit verschiedenen Buchstaben weichen signifikant voneinander ab (Games-Howell, $p<.05$ )

Tabelle 5: Verteilung der Negativität nach Land

\begin{tabular}{lccccc}
\hline Negativitätsindex (von -1 bis 1$)$ & Deutschland & Österreich & Schweiz & Gesamt \\
\hline \multirow{2}{*}{ Positiv $(<0)$} & $n$ & 125 & 88 & 131 & 344 \\
& $\%$ & 25,1 & 18,4 & 33,5 & 25,2 \\
\hline \multirow{2}{*}{ Neutral/ausgewogen $(=0)$} & $n$ & 108 & 61 & 68 & 237 \\
& $\%$ & 21,7 & 12,8 & 17,4 & 17,3 \\
\hline \multirow{2}{*}{ Negativ (> 0) } & $n$ & 265 & 328 & 192 & 785 \\
& $\%$ & 53,2 & 68,8 & 49,1 & 57,5 \\
\hline \multirow{2}{*}{ Gesamt } & $n$ & 498 & 477 & 391 & 1366 \\
& $\%$ & 100,0 & 100,0 & 100,0 & 100,0 \\
\hline
\end{tabular}

Anmerkung: $\chi^{2}(4, N=1366)=47,38, p<, 001$

\subsection{Unterschiede zwischen den Mediengattungen}

Medien berichten online signifikant negativer über Politik als offline. Dieser Befund zeigt sich beim Negativitätsindex und der Konfliktdimension (siehe Tabelle 6). Bei der Tonalität ist der Unterschied nur tendenziell signifikant, bei den übrigen Dimensionen nicht signifikant. Damit bestätigt sich Hypothese 3, allerdings nicht besonders deutlich. Schlüsselt man die Analyse zusätzlich nach Ländern auf (nicht dargestellt), bestätigen sich die Ergebnisse bis auf eine Ausnahme: In der Schweiz berichten die Medien entgegen dem Trend offline mehr über die Untauglichkeit $\left(M_{\text {offline }}=0,18\right.$ vs. $\left.M_{\text {online }}=0,13\right)$ und Unvorteilhaftigkeit $\left(M_{\text {offline }}=-0,16\right.$ vs. $\left.M_{\text {online }}=-0,11\right)$ von Politikern als offline. Diese Unterschiede sind jedoch nicht signifikant.

Beim Vergleich von Presse und TV sind die Befunde recht heterogen. Betrachtet man erneut die Mittelwerte des Negativitätsindex, zeichnet sich ab, dass das Privatfernsehen durchschnittlich am negativsten berichtet und die Boulevardzeitungen am wenigsten negativ (siehe Tabelle 7). Die Qualitätszeitungen und das öffentliche Fernsehen sind dazwischen angesiedelt. Diese Unterschiede sind jedoch nur zwischen Privatfernsehen und Boulevardzeitungen signifikant, und das auch nur für den Negativitätsindex sowie 
Tabelle 6: Negativität nach Offline-/Online

\begin{tabular}{llccc}
\hline Negativität & & Offline $(n=836)$ & Online $(n=530)$ & $t$ \\
\hline Index & 0,18 & 0,24 & $t(1364)=-2,45, p=, 015$ \\
\hline \multirow{3}{*}{ Dimension } & Tonalität & 0,21 & 0,27 & $t(1364)=-1,69, p=, 091$ \\
& Konflikt & 0,31 & 0,44 & $t(1364)=-3,46, p=, 001$ \\
& Untauglichkeit & 0,18 & 0,21 & $t(1075)=-0,95, p=, 345$ \\
& Unvorteilhaftigkeit & 0,00 & 0,03 & $t(1344)=-1,44, p=, 150$ \\
\hline
\end{tabular}

Anmerkung: Mittelwerte; Skala von -1 (= positiv) über 0 (= ambivalent/neutral) zu 1 (= negativ)

die Dimensionen Tonalität und Konflikt. Die Unvorteilhaftigkeitsdimension ist bei den Qualitätszeitungen signifikant am stärksten ausgeprägt und beim öffentlichen Fernsehen signifikant am schwächsten. Differenziert man zusätzlich nach Ländern, so berichtet in Deutschland die Bild-Zeitung $(M=0,01)$ und in der Schweiz SF1 $(M=0,02)$ am wenigsten negativ, wenngleich beides nicht signifikant.

\section{Tabelle 7: Mittelwerte der Negativität nach Mediengattung}

\begin{tabular}{|c|c|c|c|c|c|c|}
\hline \multirow{2}{*}{\multicolumn{2}{|c|}{ Negativität }} & \multicolumn{2}{|c|}{ Presse } & \multicolumn{2}{|c|}{ TV } & \multirow[b]{2}{*}{$F$} \\
\hline & & $\begin{array}{l}\text { Qualität } \\
(n=653)\end{array}$ & $\begin{array}{c}\text { Boulevard } \\
(n=286)\end{array}$ & $\begin{array}{c}\text { Öffent- } \\
\text { lich } \\
(n=255)\end{array}$ & $\begin{array}{c}\text { Privat } \\
(n=172)\end{array}$ & \\
\hline \multicolumn{2}{|l|}{ Index } & $0,22^{\mathrm{ab}}$ & $0,14^{a}$ & $0,18^{\mathrm{ab}}$ & $0,27^{b}$ & $\begin{array}{c}F(3,1362)=3,61, \\
p=, 013\end{array}$ \\
\hline \multirow{4}{*}{$\begin{array}{l}\text { Dimen- } \\
\text { sion }\end{array}$} & Tonalität & $0,23^{\mathrm{ab}}$ & $0,16^{a}$ & $0,24 \mathrm{ab}$ & $0,36^{b}$ & \multirow{4}{*}{$\begin{array}{c}F(3,1362)=3,28, \\
p=, 020 \\
F(3,1362)=3,32, \\
p=, 017 \\
F(3,1362)=1,54, \\
p=, 204 \\
F(3,1342)=4,07, \\
p=, 007\end{array}$} \\
\hline & Konflikt & $0,38^{a b}$ & $0,28^{a}$ & $0,34 \mathrm{ab}$ & $0,46^{b}$ & \\
\hline & Untauglichkeit & 0,21 & 0,14 & 0,16 & 0,23 & \\
\hline & $\begin{array}{l}\text { Unvorteilhaftig- } \\
\text { keit }\end{array}$ & $0,04^{a}$ & $-0,03^{a b}$ & $-0,04 \mathrm{~b}$ & $0,02^{\mathrm{ab}}$ & \\
\hline
\end{tabular}

Anmerkung: Skala von -1 (= positiv) über 0 (= ambivalent/neutral) zu 1 (= negativ); Werte mit verschiedenen Buchstaben weichen signifikant voneinander ab (Gabriel, Games-Howell, p <.05)

Vor diesem Hintergrund bestätigt sich Hypothese 4 nur eingeschränkt. Zum einen berichtet das Privatfernsehen zwar am negativsten, aber die Unterschiede zum öffentlichen Fernsehen sind nicht signifikant. Zum anderen berichtet das öffentliche Fernsehen bei der Unvorteilhaftigkeitsdimension und in der Schweiz am wenigsten negativ, aber auch dort sind die Unterschiede zum Privatfernsehen nicht signifikant.

Wirft man jedoch wieder einen Blick auf die Verteilung des Negativitätsindex (siehe Tabelle 8), liegt der Anteil der eher positiv gehaltenen Beiträge beim öffentlichen Fernsehen bei rund einem Viertel (25,5\%) und damit signifikant über dem Anteil des Privatfernsehens von rund einem Fünftel (19,2\%). Zugleich produziert das öffentliche Fernsehen signifikant weniger negative Beiträge (58 \%) als das Privatfernsehen (65,1\%). Diese Befunde können wiederum als Bestätigung für Hypothese 4 gewertet werden. 
Tabelle 8: Verteilung der Negativität nach Mediengattung

\begin{tabular}{|c|c|c|c|c|c|c|}
\hline \multirow{2}{*}{\multicolumn{2}{|c|}{ Negativitätsindex (von -1 bis 1 ) }} & \multicolumn{2}{|c|}{ Presse } & \multicolumn{2}{|c|}{ TV } & \multirow{2}{*}{ Gesamt } \\
\hline & & Qualität & Boulevard & Öffentlich & Privat & \\
\hline \multirow[b]{2}{*}{ Positiv $(<0)$} & $n$ & 152 & 94 & 65 & 33 & 344 \\
\hline & $\%$ & 23,3 & 32,9 & 25,5 & 19,2 & 25,2 \\
\hline \multirow{2}{*}{ Neutral/ausgewogen $(=0)$} & $n$ & 123 & 45 & 42 & 27 & 237 \\
\hline & $\%$ & 18,8 & 15,7 & 16,5 & 15,7 & 17,3 \\
\hline \multirow[t]{2}{*}{ Negativ $(>0)$} & $n$ & 378 & 147 & 148 & 112 & 785 \\
\hline & $\%$ & 57,9 & 51,4 & 58,0 & 65,1 & 57,5 \\
\hline Gesamt & $\begin{array}{l}n \\
\%\end{array}$ & $\begin{array}{c}653 \\
100,0\end{array}$ & $\begin{array}{c}286 \\
100,0\end{array}$ & $\begin{array}{c}255 \\
100,0\end{array}$ & $\begin{array}{c}172 \\
100,0\end{array}$ & $\begin{array}{l}1366 \\
1000\end{array}$ \\
\hline
\end{tabular}

Anmerkung: $\chi 2(6, \mathrm{~N}=1366)=15,38, \mathrm{p}<, 018$

Die Boulevardpresse verfasst die meisten positiven (32,9\%) und die wenigsten negativen $(51,4 \%)$ Beiträge unter allen Mediengattungen. Daher lässt sich bezüglich der Forschungsfrage 1 festhalten, dass die Boulevardzeitungen nicht nur durchschnittlich am wenigsten negativ berichten. Sie bringen auch die meisten positiven und die wenigsten negativen Beiträge.

\subsection{Unterschiede zwischen Darstellungsformen und Themen}

Auf Beitragsebene sind die meinungsbetonten Darstellungsformen Kommentar und Leitartikel signifikant negativer gehalten als die übrigen Formen (siehe Tabelle 9). Bei der Tonalität erreichen sie sogar Indexwerte von $M=0,49$. Damit bestätigt sich Hypothese 5. Interview und Portrait sind dagegen die am wenigsten negativen Darstellungsformen, wobei letzteres sogar einen Indexwert aufweist, der in der unmittelbaren Nähe von Ausgewogenheit oder Neutralität liegt. Allerdings werden diese Unterschiede aufgrund der geringen Fallzahlen nicht signifikant. Bei Tonalität $(M=-0,13)$ und Unvorteilhaftigkeit $(M=-0,17)$ befindet sich das Portrait sogar deutlich im Bereich positiver Berichterstattung. Dieser Befund ist aus zwei Gründen nachvollziehbar: Erstens erhalten Journalisten in der Regel keine Autorisierung für ein Interview oder Portrait, das die dargestellte Person überwiegend in schlechtem Licht erscheinen lässt oder eine negative Gesamtstimmung erzeugt. Zweitens hält sich häufig auch die dargestellte Person mit allzu kritischen oder despektierlichen Aussagen zurück, da diese auf sie selbst zurückfallen könnten.

\section{Tabelle 9: Negativität nach Darstellungsform}

\section{Darstellungsform}

Kommentar/Leitartikel $(n=108)$

Bericht $(n=1020)$

Reportage/Hintergrundbericht $(n=144)$

Interview $(n=58)$

Portrait $(n=15)$

Sonstiges $(n=21)$
Negativitätsindex $(M)$

$0,38^{a}$
$0,19^{b}$
$0,18^{b}$
$0,10^{b}$
$0,01^{b}$
$0,06^{b}$

$0,38^{a}$

$0,18^{b}$

$0,10^{b}$

$0,06^{b}$

Anmerkung: Skala von -1 (= positiv) über 0 (= ambivalent/neutral) zu 1 (= negativ); $F(5,1360)=$ $5,16, p<, 001$; Werte mit verschiedenen Buchstaben weichen signifikant voneinander ab (Gabriel, Games-Howell, $p<.05$ ) 
Vornehmlich negativ wird berichtet über Themen wie (Nicht-)Funktionieren der Demokratie, Skandale, Kriminalität, Probleme von Unternehmen und Konsumenten, Immigration, bezahlbare Wohnungen und Verkehr, Arbeitslosigkeit sowie innere Sicherheit und Terror (siehe Tabelle 10). Bei den besonders negativ präsentierten Themen Funktionieren der Demokratie sowie Justiz und Kriminalität tragen die Dimensionen Konflikt und Tonalität am meisten zu den hohen Indexwerten bei. Dies dürfte zum einen an ihrer Verknüpfung zu negativ konnotierten Ereignislagen und zum anderen an ihrem Auslösepotenzial für kognitive Mobilisierungseffekte liegen. Einerseits erfüllt eine solche Prioritätensetzung die gesellschaftliche Warnfunktion der Medien, andererseits bedient sie den kommerziell orientierten Animationsnutzen der Berichterstattung. Gerade in Österreich erfüllte eine Reihe medial intensiv beachteter politischer Skandale die Funktion als „situational triggers“ für eine entsprechend dieser Motivlage negativ geladene Berichterstattung. ${ }^{4}$

Über Vermischtes, Sport und Umwelt wird am wenigsten negativ berichtet. Für diese Themen liegen die Indexwerte sogar im Bereich der positiven Berichterstattung. Dies lässt sich zumindest teilweise damit erklären, dass beim Sport das positive Erlebnis und der Erfolg im Zentrum stehen. Zum niedrigen Indexwert des Umweltthemas trägt in erster Linie die außergewöhnlich schwach ausgeprägte Unvorteilhaftigkeitsdimension $(M=-0,22)$ bei. Offenbar genießen politische Akteure im Umweltbereich besondere Sympathie. Bezüglich Forschungsfrage 2 lässt sich festhalten, dass über normativ aufgeladene Themen eher negativ und über „weichere“ Themen (z. B. Sport, Umwelt, Kultur) eher weniger negativ berichtet wird.

\subsection{Multivariates Regressionsmodell}

Die wichtigsten Faktoren der Länder-, Medien- und Beitragsebene wurden zur gegenseitigen Kontrolle und Quantifizierung der Einflüsse in ein multivariates Regressions-

4 In Österreich wurde im Untersuchungszeitraum ein parlamentarischer Untersuchungsausschuss eingerichtet, der sich mit der im Jahr 2003 von der Regierung in Auftrag gegebenen Vergabe des Polizeifunks beschäftigte. Eine Reihe an ehemaligen Spitzenpolitikern sowie Vertreter aus der Wirtschaft wurden hierzu befragt und unrechtmäßige Verhaltensweisen wurden diskutiert. Zusätzlich wurde im Untersuchungsausschuss die sog. BUWOG-Affäre behandelt. Dem ehemaligen Finanzminister Karl-Heinz Grasser wurden u. a. Untreue, illegale Absprachen und Provisionszahlungen in Zusammenhang mit der Privatisierung von 60.000 Bundeswohnungen vorgeworfen. Zur gleichen Zeit wurde in den Medien der Prozess gegen den ehemaligen EU-Abgeordneten Ernst Strasser dargestellt, der sich dem Vorwurf der Korruption stellen musste. Ebenfalls zu dieser Zeit wurde gegen mehrere Mitglieder des Landtags in Kärnten aufgrund schwerwiegender Korruptionsvorwürfe im Zusammenhang mit der Bank Hypo Alpe Adria ermittelt. Ein Spitzenpolitiker der Freiheitlichen Partei Kärnten (FPK), die in Kärnten die politische Mehrheit bildete, musste sich zudem wegen Vorwürfen der Untreue vor Gericht verantworten. Die daraufhin von der Kärntner Opposition angestrebten Neuwahlen wurden dann von der amtierenden FPK-Mehrheit durch Sondersitzungen blockiert. Im selben Zeitraum wurden Vorwürfe gegen den zweiten Nationalratspräsidenten Martin Graf erhoben, sich an einer Privatstiftung bereichert zu haben. 
Tabelle 10: Negativität nach Thema

\section{Thema}

Funktionieren der Demokratie, Regierungsqualität, Skandale $(n=177)$

Justiz, Kriminalität $(n=83)$

Betriebswirtschaft: Unternehmen, Konsumenten $(n=17)$

Immigration, Integration $(n=51)$

Verkehr: öffentlicher und privater $(n=89)$

Arbeitslosigkeit und Arbeitsmarktpolitik $(n=22)$

Wohnen, Bau, Stadtentwicklung $(n=21)$

Verteidigung, Sicherheit, Terror $(n=62)$

Landwirtschaft $(n=8)$

Rechte und Freiheiten der Bürger $(n=57)$

Parteipolitik, Parteiquerelen, Wahlen $(n=176)$

Volkswirtschaft: Steuern, Haushalt, Finanzkrise $(n=190)$

Soziales: Gesundheit, Renten $(n=96)$

Kultur, Medien, Kommunikation $(n=28)$

Außenpolitik, EU $(n=102)$

Bildung $(n=46)$

Umwelt $(n=53)$

Vermischtes, Sport $(n=88)$
Negativitätsindex $(M)$

\section{0,46}

0,45

0,27

0,26

0,25

0,24

0,22

0,21

0,20

0,19

0,16

0,15

0,11

0,10

0,09

0,09

$-0,03$

$-0,05$

Anmerkung: Skala von -1 (= positiv) über 0 (= ambivalent/neutral) zu 1 (= negativ); $F(17,1348)=$ $9,61, p<, 001$

\section{Tabelle 11: Einflussfaktoren der Negativität}

\begin{tabular}{|c|c|c|c|}
\hline Ebene & Prädiktor & Beta & $\Delta R^{2}$ \\
\hline Land $^{a}$ & $\begin{array}{l}\text { Österreich } \\
\text { Schweiz }\end{array}$ & $\begin{array}{l}14 * * * \\
, 02\end{array}$ &, $05 * * *$ \\
\hline Medium $^{b}$ & $\begin{array}{l}\text { Onlinemedium } \\
\text { Privatfernsehen } \\
\text { Öffentliches Fernsehen } \\
\text { Boulevardpresse }\end{array}$ & $\begin{array}{l}, 06 \% \\
, 03 \\
-, 03 \\
-, 04\end{array}$ &, $01 * *$ \\
\hline Beitrag & $\begin{array}{l}\text { Thema: Funktionieren der Demokratie } \\
\text { Thema: Justiz/Kriminalität } \\
\text { Kommentar/Leitartikel } \\
\text { Thema: Umwelt } \\
\text { Thema: Vermischtes/Sport }\end{array}$ & $\begin{array}{l}, 18 * * * \% \\
, 15 * \% * \\
, 10 * * \% \\
-, 07 * * \\
-, 10 * * \%\end{array}$ &, $08^{* * * * *}$ \\
\hline
\end{tabular}

Anmerkung: Negativitätsindex als abhängige Variable; Skala von -1 (= positiv) über 0 (= ambivalent/ neutral) zu 1 (= negativ); $R^{2}=, 13, F(11,1354)=18,77, p<, 001$; markierte Koeffizienten sind signifikant $(* \mathrm{p}<, 05 ; * \mathrm{p}<, 01 ; * * \mathrm{p}<, 001)$

a „Deutschland“ als Referenzkategorie; b "Qualitätspresse“ als Referenzkategorie 
modell integriert (siehe Tabelle 11). ${ }^{5}$ Die Ergebnisse dieser Analyse sind aufgrund der Mehrebenenstruktur der Daten und der dichotomen Prädiktoren mit Vorsicht zu interpretieren und sollen nur zur zusätzlichen Orientierung dienen.

Das Modell erklärt insgesamt rund $13 \%$ der Varianz beim Negativitätsindex. Die größten Einflüsse finden sich auf der Beitragsebene, die rund acht Prozentpunkte zur Varianzaufklärung beiträgt. Während über die Themen Funktionieren der Demokratie ( $3 \%$ Varianzaufklärung), Justiz und Kriminalität (2\%) sowie in meinungsbetonten Darstellungsformen (1\%) signifikant negativer berichtet wird, wird über die Themen Vermischtes, Sport und Umwelt (je $1 \%$ ) signifikant weniger negativ berichtet. Auf die Länderebene entfallen immerhin noch rund 5 Prozentpunkte Varianzaufklärung: Medien aus Österreich berichten signifikant negativer über Politik (2\%). Die Medienebene trägt lediglich einen Prozentpunkt Varianzaufklärung bei und beherbergt einen Faktor mit signifikantem Einfluss: Medien berichten online negativer $(<1 \%)$.

Die auf bivariaten Analysen beruhenden Befunde, dass Medien in Österreich (H1), im Online-Format (H3) und in meinungsbetonten Darstellungsformen (H5) signifikant negativer über Politik berichten, bleiben auch im Regressionsmodell bestehen, wodurch sich die entsprechenden Hypothesen weiter erhärten. Auch die Schlussfolgerungen zur Forschungsfrage 2, wonach über normativ aufgeladene Themen signifikant negativer und über „weiche“ Themen signifikant positiver berichtet wird, werden untermauert. Allerdings sind die Effektstärken allesamt sehr klein und die partielle Varianzaufklärung der einzelnen Faktoren bewegt sich im Bereich von ein bis drei Prozent. Außerdem finden sich in der multivariaten Analyse keine zusätzlichen Hinweise zur Bestätigung von $\mathrm{H} 2$ (geringere Negativität in der Schweiz) und H4 (geringere Negativität beim öffentlichen Fernsehen) sowie keine weiteren Antworten auf Forschungsfrage $1 \mathrm{zu}$ den Unterschieden zwischen Qualitäts- und Boulevardpresse.

\section{Diskussion und Fazit}

Die Hypothesen, dass Medien in Österreich (H1), online (H3) und in meinungsbetonten Darstellungsformen (H5) signifikant negativer über Politik berichten als in ihren Vergleichsgruppen, wurden durch die empirischen Analysen bestätigt. Allerdings konnte bei der ersten Hypothese nicht geklärt werden, ob das höhere Negativitätsniveau in Österreich auf eine kompetitivere Kommunikationskultur, eine kritischere journalistische Kultur oder die durch politische Skandale bestimmte Ereignislage im Untersuchungszeitraum zurückzuführen ist.

Die dritte Hypothese bestätigte sich nur für den Gesamtindex und die Konfliktdimension. In der Schweiz berichteten die Medien teilweise sogar offline negativer als online. ${ }^{6}$ Die fünfte Hypothese wurde vollumfänglich bestätigt. Zusätzlich stellte sich heraus, dass Interviews und Portraits durch eine relativ geringe Negativität gekennzeichnet sind.

5 Auf Beitragsebene wurden die Faktoren berücksichtigt, die in den bivariaten Analysen für die größten Abweichungen des Negativitätsindex verantwortlich waren. Bei den Darstellungsformen machte lediglich die Kategorie Kommentar/Leitartikel einen signifikanten Unterschied. Da bei den Themen aufgrund der zahlreichen Untergruppen keine signifikanten Unterschiede vorkamen, wurden die Themen ausgewählt, die die deutlichsten Abweichungen nach oben und unten bewirkten.

6 In einer parallel zur vorliegenden Untersuchung durchgeführten Analyse stellte sich heraus, dass auch andere westliche Länder offline negativer berichten als online (de Vreese, Esser \& Hopmann i. E.). 
Die zwei übrigen Hypothesen ( $\mathrm{H} 2$ und $\mathrm{H} 4)$ konnten nicht uneingeschränkt beibehalten werden. Schweizerische Medien berichten entgegen der zweiten Hypothese durchschnittlich nicht weniger negativ als deutsche Medien, sie bringen jedoch mehr positiv gehaltene Beiträge und stellen politische Akteure seltener unvorteilhaft dar. An diesen Stellen scheint sich die von Lijphart (2012) postulierte „freundlichere und sanftere" Qualität der Konsensdemokratie in der öffentlichen Kommunikation niederzuschlagen. Das öffentliche Fernsehen produziert im Einklang mit der vierten Hypothese zwar durchschnittlich weniger negative Beiträge als das Privatfernsehen, doch diese Unterschiede sind nicht signifikant. Sie werden dies erst, wenn man die Anteile an negativen und positiven Beiträgen betrachtet. Es ist aufschlussreich und deckt sich wiederum mit der zweiten Hypothese, dass das öffentliche Fernsehen in der Schweiz im Vergleich zu den Mediengattungen am wenigsten negativ berichtet.

Hinsichtlich der ersten Forschungsfrage offenbarte sich entgegen den gängigen Erwartungen, dass die Boulevardpresse unter allen Mediengattungen sowohl am wenigsten negativ als auch am positivsten schreibt, allen voran die Bild-Zeitung in Deutschland. Hier bestätigen sich die eingangs erwähnten Befunde von Eilders (1997) und anderen Autoren zur relativ konfliktreichen Berichterstattung der Qualitätszeitungen. Die weit verbreitete Vorstellung, dass sich die allgemeine Tendenz des Boulevardjournalismus zum Sensationalismus zwangsläufig in hohen Negativitätsniveaus niederschlägt, muss wohl hinterfragt werden. Es wird vielmehr ersichtlich, dass "gute“ Nachrichten in der Boulevardpresse einen festen Stellenwert haben. Vermutlich werden diese sogar gezielt zur Abgrenzung gegenüber der Qualitätspresse eingesetzt. Die dahinterstehende Argumentation könnte lauten, dass man den Leser nicht durch übermäßige Kritik vergraulen und ihm stattdessen auch Lichtblicke bieten möchte.

Die Beschäftigung mit der zweiten Forschungsfrage zeigte, dass über normativ aufgeladene Themen stärker negativ berichtet wird und über „weiche“ Themen weniger negativ. Doch auch hier blieb unklar, ob diese Unterschiede mit der generellen Beschaffenheit dieser Themen oder den Ereignissen im Untersuchungszeitraum zusammenhängen. Insgesamt stellte sich heraus, dass die Negativität der Politikberichterstattung stärker durch Thema und Form des einzelnen Beitrags bestimmt wird als durch das dahinterstehende Medium oder Land. Auch die relativ geringe Erklärungskraft des multivariaten Modells legt nahe, dass die Negativitätsschwankungen stark der aktuellen Ereignislage unterliegen.

Die vorliegende Untersuchung unterliegt einigen Beschränkungen, aus denen sich Anregungen für weiterführende Studien ableiten lassen. Erstens war das Ansinnen, Routineperioden zu untersuchen, nur bedingt erfolgreich. Auch außerhalb der Wahlkämpfe ist die Chance bei international vergleichenden Untersuchungen hoch, dass besondere Ereignisse die nationalen Themenagenden verzerren. Ein Mittel dagegen könnte die Ausdehnung des Untersuchungszeitraums auf ein ganzes Jahr (oder noch weiter) darstellen.

Zweitens kann die traditionelle Konzentration auf die Negativität dazu verleiten, positive Berichterstattung lediglich als „die andere Seite der Medaille“ zu betrachten oder sogar vollständig zu vernachlässigen. Die vorliegende Untersuchung konnte jedoch insbesondere am Fall der Schweiz und der Boulevardpresse - zeigen, dass der „Positivität" durchaus eine eigene Qualität innewohnen kann. Diese Tatsache nimmt auch die Wirkungsforschung zunehmend zur Kenntnis (z. B. Schuck \& de Vreese 2012). Daher plädiert dieser Beitrag für eine Intensivierung der Positivitätsforschung in der Zukunft.

Drittens gibt es angesichts der vorliegenden Untersuchung Grund zur Annahme, dass sowohl eine kritische als auch eine sensationalistische Spielart der Negativität existieren. 
Während erstere vermutlich in der Qualitätspresse und dem öffentlichen Fernsehen dominiert, kommt letztere vor allem in der Boulevardpresse und dem Privatfernsehen vor. Dies könnte auch das hohe Negativitätsniveau bei den Qualitätszeitungen und die heterogenen Unterschiede zwischen den Mediengattungen erklären. Allerdings ist es uns trotz relativ aufwändiger Operationalisierung nicht gelungen, zwischen diesen beiden Typen der Negativität zu unterscheiden. Dies könnte eine Herausforderung für weiterführende Studien darstellen.

Viertens beschränkt sich die vorliegende Untersuchung auf den deutschsprachigen Raum. Zur weiteren Kontextualisierung und Generalisierung der Befunde wäre es erforderlich, die Länderstichprobe auszuweiten, insbesondere mit Blick auf Unterschiede im Mediensystem und politischen System. Diesem Ansinnen geht eine Folgestudie von uns nach (de Vreese, Esser \& Hopmann i. E.).

\section{Literatur}

Baumeister, R., Bratslavsky, E., Finkenhauer, C. \& Vohs, K. (2001). Bad is Stronger than Good. Review of General Psychology, 5(4), 323-70.

Bennett, L. W. (2012). News: The Politics of Illusion (9. Aufl.). New York: Pearson.

Blumler, J. \& Gurevitch, M. (1995). Comparative Research: The Extending Frontier. In J. Blumler $\&$ M. Gurevitch (Hrsg.), The Crisis of Public Communication (S. 73-85). London: Routledge.

de Swert, K., Belo, A., Kamhawi, R., Lo, V., Mujica, C. \& Porath, W. (2013). Topics in Foreign and Domestic Television News. In A. Cohen (Hrsg.), Foreign News on Television. Where in the World is the Global Village? (S. 41-62). New York: Lang.

de Vreese, C., Esser, F. \& Hopmann, D. (i. E.). Where Is the Good News? Comparing Political Online and Offline Journalism in 16 Countries. Buchmanuskript.

Eilders, C. (1997). Nachrichtenfaktoren und Rezeption: Eine empirische Analyse zur Auswabl und Verarbeitung politischer Information. Opladen: Westdeutscher Verlag.

Esser, F. \& Hanitzsch, T. (2012). On the Why and How of Comparative Inquiry in Communication Studies. In F. Esser \& T. Hanitzsch (Hrsg.), Handbook of Comparative Communication Research (S. 3-22). London: Routledge.

Esser, F. \& Hemmer, K. (2008). Characteristics and Dynamics of Election News Coverage in Germany. In J. Strömbäck \& L. L. Kaid (Hrsg.), The Handbook of Election News Coverage around the World (S. 289-307). New York: Routledge.

Esser, F. \& Pfetsch, B. (2003). Gut, dass wir verglichen haben: Bilanz und Bedeutung der komparativen politischen Kommunikationsforschung. In F. Esser \& B. Pfetsch (Hrsg.), Politische Kommunikation im internationalen Vergleich: Grundlagen, Anwendungen, Perspektiven (S. 437-494). Opladen: Westdeutscher Verlag

Galtung, J. \& Ruge, M. H. (1965). The Structure of Foreign News: The Presentation of the Congo, Cuba and Cyprus Crisis in Four Norwegian Newspapers. Journal of Peace Research, 2(1), 64-91.

Hanitzsch, T. \& Berganza, R. (2012). Explaining Journalists' Trust in Public Institutions across 20 Countries: Media Freedom, Corruption and Ownership Matter Most. Journal of Communication, 62(5), 794-814.

Hanitzsch, T., Hanusch, F., Mellado, C., Anikina, M., Berganza, R. \& Cangoz, I. (2011). Mapping Journalism Cultures across Nations. Journalism Studies, 12(3), 273-293.

Kahneman, D. \& Tversky, A. (1979). Prospect Theory: An Analysis of Decision under Risk. Econometrics, 47, 111-132.

Kaltenbrunner, A., Karmasin, M., Kraus, D. \& Zimmermann, A. (2008). Der Journalisten-Report II: Österreichs Medienmacher und ihre Motive: Eine repräsentative Befragung. Wien: Facultas.

Kepplinger, H. M. \& Weissbecker, H. (1991). Negativität als Nachrichtenideologie. Publizistik, 36(3), 330-342.

Kepplinger, H. M. (2010). Medieneffekte: Theorie und Praxis öffentlicher Kommunikation. Wiesbaden: VS. 
Kleinnijenhuis, J. (2008). Negativity. In W. Donsbach (Hrsg.), The International Encyclopedia of Communication (S. 3188-3192). Malden, MA: Blackwell.

Klingemann, H. D. (2013). Dissatisfied Democrats: Evidence from Old and New Democracies. In R. J. Dalton \& C. Welzel (Hrsg.), The Civic Culture Revisited: From Allegiant to Assertive Citizens (o. S.). Cambridge: Cambridge University Press.

Lengauer, G., Esser, F. \& Berganza, R. (2012). Negativity in Political News: A Review of Concepts, Operationalizations and Key Findings, Journalism, 13(2), 179-202.

Lijphart, A. (2012). Patterns of Democracy: Government Forms and Performance in Thirty-Six Countries. New Haven: Yale University Press.

Magin, M. (2012). Wablkampf in Deutschland und Österreich: Ein Langzeitvergleich der Presseberichterstattung (1949-2006). Köln: Böhlau.

Maurer, M. (2003). Politikverdrossenheit durch Medienberichte: Eine Panelanalyse. Konstanz: UVK.

Maurer, M. \& Reinemann, C. (2006). Medieninhalte: Eine Einführung. Wiesbaden: VS.

Norris, P. (2000). A Virtuous Circle: Political Communication in Postindustrial Societies. Cambridge: Cambridge University Press.

O’Neill, D. \& Harcup, T. (2009). News Values and Selectivity. In K. Wahl-Jorgensen \& T. Hanitzsch (Hrsg.), The Handbook of Journalism Studies (S. 161-174). New York: Routledge.

Oschatz, C., Maurer, M. \& Haßler, J. (2014). (R)Evolution der Politikberichterstattung im Medienwandel. Medien E Kommunikationswissenschaft, 62(1), 25-41.

Patterson, T. E. (1996). Bad News, Bad Governance. The ANNALS of the American Academy of Political and Social Science, 546(1), 97-108.

Patterson, T. E. (2002). The Vanishing Voter: Public Involvement in an Age of Uncertainty. New York: Knopf.

Plasser, F., Pallaver, G. \& Lengauer, G. (2009). Die (trans-)nationale Nachrichtenlogik in Mediendemokratien: Politischer TV-Journalismus im Wahlkampf zwischen transatlantischer Konvergenz und nationaler Divergenz. In F. Marcinkowski \& B. Pfetsch (Hrsg.), Politik in der Mediendemokratie (S. 174-202). Wiesbaden: VS.

Powers, M. \& Benson, R. (2014). Is the Internet Homogenizing or Diversifying the News? External Pluralism in the U.S., Danish, and French Press. International Journal of Press/Politics, 19(2), 246-265.

Przeworski, A. \& Teune, H. J. (1970). The Logic of Comparative Social Inquiry. New York: Wiley.

Quandt, T. (2008). Neues Medium. alter Journalismus? Eine vergleichende Inhaltsanalyse tagesaktueller Print- und Online-Nachrichtenangebote. In T. Quandt \& W. Schweiger (Hrsg.), Journalismus online: Partizipation oder Profession (S. 131-155). Wiesbaden: VS.

Reinemann, C., Maurer, M., Zerback, T. \& Jandura, O. (2013). Die Spätentscheider: Medieneinflüsse auf kurzfristige Wablentscheidungen. Wiesbaden: VS.

Ridout, T. \& Franz, M. (2008). Evaluating Measures of Campaign Tone. Political Communication, 25(2), 158-179.

Ruhrmann, G., Woelke, J., Maier M. \& Diehlmann, N. (2003). Der Wert der Nachrichten im deutschen Fernsehen: Ein Modell zur Validierung der Nachrichtenfaktoren. Opladen: Leske und Budrich.

Schönbach, K. (1977). Trennung von Nachricht und Meinung: Empirische Untersuchung eines journalistischen Qualitätskriteriums. Freiburg: Alber.

Schuck, A. \& de Vreese, C. H. (2012). When Good News is Bad News: Explicating the Moderated Mediation Dynamic behind the Reversed Mobilization Effect. Journal of Communication, 62(1), 57-77.

Shoemaker, P. \& Cohen, A. A. (2006). News Around the World: Content, Practitioners, and the Public. New York: Routledge.

Strömbäck, J. \& Kaid, L. L. (2008). A Framework for Comparing Election News Coverage around the World. In J. Strömbäck \& L. L. Kaid (Hrsg.), The Handbook of Election Nerws Coverage around the World (S.1-18). New York: Routledge.

Takens, J., van Atteveldt, W., van Hoof, A. \& Kleinnijenhuis, J. (2013). Media Logic in Election Campaign Coverage. European Journal of Communication, 28(3), 277-293. 
Transparency International (2012). Corruption Perceptions Index 2012, http://www.transparen cy.org/cpi2012/results\#myAnchor1 [08.10.2014].

Walter, A. S. \& Vliegenthart, R. (2010). Negative Campaigning across Different Communication Channels: Different Ballgames? International Journal Press/Politics, 15(4), 441-461.

Weiß, H.-J. (1982). Die Wahlkampfberichterstattung und -kommentierung von Fernsehen und Tagespresse zum Bundestagswahlkampf 1980. Media Perspektiven, 4, 263-275.

Westen, D. (2007). The Political Brain: The Role of Emotion in Deciding the Fate of the Nation. New York: Public Affairs.

Wirth, W. \& Kolb, S. (2003). Äquivalenz als Problem: Forschungsstrategien und Designs der komparativen Kommunikationswissenschaft. In F. Esser \& B. Pfetsch (Hrsg.), Politische Kommunikation im internationalen Vergleich (S. 105-131). Opladen: Westdeutscher Verlag.

Wolling, J. (1999). Politikverdrossenheit durch Massenmedien? Der Einfluss der Medien auf die Einstellungen der Bürger zur Politik. Opladen/Wiesbaden: Westdeutscher Verlag.

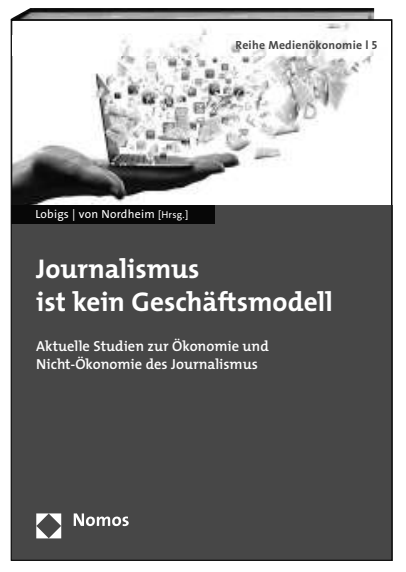

\section{Journalismus} ist kein Geschäftsmodell

Aktuelle Studien zur Ökonomie und Nicht-Ökonomie des Journalismus

Herausgegeben von Frank Lobigs und Gerret von Nordheim

2014, 269 S., brosch., 46,- $€$

ISBN 978-3-8487-1150-5

(Reihe Medienökonomie, $B d .5$ )

www.nomos-shop.de/22260

Journalismus und Ökonomie sind zwar getrennt zu sehen, doch stehen sie in einer schicksalhaften Verbindung. Die Studien in diesem Band prüfen den Status quo dieser Verbindung mit teils erstaunlich positiven Resultaten, die auf Potenziale der Medienregulierung und des Medienmanagements verweisen, um auch künftig die Autonomie des Journalismus zu bewahren.

Bestellen Sie jetzt telefonisch unter 07221/2104-37 Portofreie Buch-Bestellungen unter www.nomos-shop.de Alle Preise inkl. Mehrwertsteuer

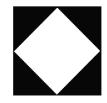

Nomos 